'Departamento de Prevención y Salud Pública Odontológica, Facultad de Odontología, Universidad de Concepción. Concepción, Chile.

${ }^{2}$ Fundación Kimntrum. Concepción, Chile. ${ }^{3}$ Universidad Andrés Bello. Concepción, Chile. ${ }^{4}$ Universidad Autónoma de Chile. Temuco, Chile. ${ }^{a}$ Cirujano Dentista, Magíster en Odontología Pediátrica.

${ }^{b}$ Cirujano Dentista, Psicólogo, Magíster en Salud Pública, Doctor en Ciencias Médicas, Magíster en Filosofía (c), Interno de Medicina

Fuente de apoyo financiero: CONICYT FONDEF FONIS + MINSAL / XV CONCURSO DE INVESTIGACIÓN Y DESARROLLO EN SALUD, FONIS 2018 SA18I0116.

Los autores declaran no tener conflictos de interés.

Recibido el 4 de enero de 2018, aceptado el 28 de marzo de 2019

Correspondencia a: Valeria Campos

Departamento de Prevención y Salud Pública Odontológica,

Facultad de Odontología, Universidad de Concepción

Avenida Roosevelt \#1550. valeriacamposcannobbio@gmail.com

\section{Estado actual de la atención sanitaria de personas con discapacidad auditiva y visual: una revisión breve}

\author{
VALERIA CAMPOS ${ }^{1,2, a}$, RICARDO CARTES-VELÁSQUEZ ${ }^{3,4, b}$
}

\section{Health care of people with visual or hearing disabilities}

Visual or hearing disabilities account for $20 \%$ of people reporting some sort of disability. We performed a literature review about the interaction that people with visual or hearing disabilities have with the health care system. We found that these people report a lower quality of life and have a higher frequency of physical and psychological ailments. They also have difficulties to obtain an adequate health care and report a paucity of trained professionals to take care of them.

(Rev Med Chile 2019; 147: 634-642)

Key words: Blindness; Health Services for Persons with Disabilities; Hearing Loss; Persons With Hearing Impairments; Vision, Low.
L as personas en situación de discapacidad (PeSD) representan a 15\% de la población mundial y a $20 \%$ de los chilenos ${ }^{1-3}$. Las discapacidades de origen sensorial, auditiva y visual, son dos de las más prevalentes en el mundo ${ }^{4}$. Existen más de 360 millones de PeSD auditiva ${ }^{1}$, afectando a $1,4 \%$ de los niños entre 5 y 14 años, y a $10 \%$ en la población mayor de 15 años $^{5}$. En Chile representa a $8,2 \%$ de las $\mathrm{PeSD}^{3}$. Por otro lado, existen 253 millones de PeSD visual, de los cuales 36 millones son legalmente ciegos ${ }^{2,6}$. A pesar de su disminución, esta condición afecta a 2,7\% de la población de Latinoamérica ${ }^{7}$. En Chile representa a $11,9 \%$ de las $\mathrm{PeSD}^{3}$.

La mayoría de las PeSD visual y auditiva provienen de un bajo nivel socioeconómico, con un menor ingreso familiar, de educación y empleo que sus contrapartes sin discapacidad ${ }^{6-11}$. Recientemente se ha evidenciado que la discapacidad visual y auditiva son responsables de la pérdida de 34 millones de AVAD (años de vida ajustados por discapacidad $)^{4}$. La mayoría de las PeSD se ven afectadas por situaciones de inequidad social y sanitaria ${ }^{8,9,11,12}$. Siendo uno de los grupos más afectados las PeSD auditivas y visuales, debido a aspectos comunicacionales ${ }^{13,14}$.

Las PeSD auditiva moderada a profunda, con una adquisición peri o prelocutiva, en su mayoría se autodenominan "Sordos" con "S" mayúscula. Ellas se definen como miembros de un mundo con su propia lengua y cultura, valores y costumbres. Esta lengua difiere del lenguaje oral en cuanto a gramática, no es universal y es considerado el elemento cultural más significativo de la comunidad Sorda ${ }^{5,15}$. Debido al proceso fonológico y falta de acceso a educación, la mayoría son analfabetas funcionales ${ }^{8,16,17}$. Las PeSD visual utilizan diversas herramientas para la lectoescritura, en general, las personas ciegas congénitas utilizan el sistema Braille, lectores de pantalla y reconocimiento de $\mathrm{voz}^{18}$. Las personas con baja visión utilizan magnificadores ópticos o electrónicos, prismas, lectores de pantalla y reconocimiento de $\mathrm{voz}^{14,18-20}$. El sistema de lectoescritura Braille no es un idioma, sino un 
alfabeto táctil que representa a la lengua oral ${ }^{21}$. Cabe destacar que la LS y el Braille son reconocidos nacional e internacionalmente ${ }^{22,23}$.

Dada las particularidades que tiene las PeSD visual y auditiva, se hace necesario que los profesionales sanitarios conozcan algunos aspectos básicos de la atención de esta población. El objetivo de esta revisión es reportar los hallazgos más relevantes reportados en la literatura de corriente principal respecto a la interacción de este grupo de personas con el sistema sanitario.

\section{Material y Métodos}

Se realizó una revisión sistemática exploratoria (Scoping review). Para efectos prácticos y siguiendo la nomenclatura utilizada en la ley chilena, a lo largo del texto nos referiremos a personas con discapacidad $(\mathrm{PcD})$ como sinónimo de PeSD.

Las bases de datos fueron seleccionadas de acuerdo a su cobertura en las disciplinas biomédicas, así las siguientes bases de datos fueron incluidas: Web of Science, LILACS, SciELO, MEDLINE, Scopus, EMBASE y Redalyc. Inicialmente no se consideraron límites en cuanto a fecha, idioma, tipo de artículo, país u otro filtro.

La pregunta de investigación fue “¿Cuáles son los principales hallazgos reportados en la literatura respecto a la salud de las personas con discapacidad auditiva $y /$ visual?".

Las palabras clave utilizadas fueron: Discapacidad auditiva, ceguera, personas con discapacidad, discapacidad sensorial, Comunidad Sorda, Sordera, discapacidad visual, limitación visual, acceso a salud, profesional de la salud, inequidad, servicios de salud, atención ambulatoria.

La búsqueda realizada fue adaptada en un algoritmo de acuerdo a los requerimientos de cada base de datos. Una segunda búsqueda incluyó la técnica de "bola de nieve" a partir de las referencias de los primeros artículos seleccionados para aumentar el número de artículos relevantes. Este proceso fue repetido una vez más para los artículos incluidos en la segunda búsqueda.

Finalmente, se seleccionaron artículos completos publicados en revistas revisadas por pares desde enero de 2004 a enero de 2019; indexadas en las bases mencionadas; en español, portugués e inglés, con metodología cuantitativa y cualitativa, se excluyeron Cartas al Editor, Reporte de casos,
Comentarios y Editoriales. Se obtuvo un total de 70 artículos.

\section{Resultados}

\section{Indicadores de salud}

Las PcD sensorial, en general reportan una menor calidad de vida que sus contrapartes sin discapacidad ${ }^{24,25}$.

\section{Comorbilidades físicas $P c D$ visual}

Las personas ciegas tienen mayores probabilidades de reportar una salud de regular a deficiente y dificultades para caminar. Por otro lado, se encuentran más propensas a presentar comorbilidades físicas que sus contrapartes sin discapacidad como esclerosis múltiples ${ }^{26}$ diabetes $^{26,27}$, hipertensión $^{26,27}$, enfermedad renal crónica ${ }^{26}$, problemas $\operatorname{cardíacos}^{26,27}$ y respiratorios ${ }^{27}$, pero sin diferencia significativa en cuanto a prevalencia de migraña y bronquiectasia. Además, se ha reportado que la combinación de discapacidad visual y diabetes en adultos mayores aumenta el riesgo de afectar negativamente el funcionamiento, participación, y estado de salud del individuo que el tener cualquiera de las condiciones por separado ${ }^{27}$.

\section{Comorbilidades físicas $P c D$ auditiva}

Se reportan escasos estudios respecto a las comorbilidades físicas de las PcD auditiva. Las personas Sordas, que utilizan la LS para comunicarse, tienen mayores tasas de obesidad y de hipertensión, pero sin diferencias en cuanto a la prevalencia de diabetes o enfermedad cardiovascular $^{28}$. Además, la discapacidad auditiva ha sido asociada de manera independiente con artritis, cáncer, enfermedades cardiovasculares, enfisema, hipertensión e infarto ${ }^{29}$. También se reporta un mayor riesgo de traumatismo en niños con discapacidad auditiva en comparación con los niños sin esta discapacidad ${ }^{30}$. Finalmente, esta discapacidad se ha asociado a un mayor número de hospitalizaciones y días de pobre salud física ${ }^{31}$.

\section{Comorbilidades psicológicas en PcD visual}

Las $\mathrm{PcD}$ visual tienen más probabilidades de tener ansiedad, angustia psicológica, depresión $y$ demencia ${ }^{26,27,32}$. Sobre el $50 \%$ se encuentran en riesgo de depresión leve a moderada ${ }^{27}$. También reportan mayores dificultades para salir de compras o sociabilizar, a diferencia de adultos sin 
discapacidad ${ }^{27}$. Además, se ha asociado la discapacidad visual con síntomas depresivos severos y mayor probabilidad de ser más fumadores, físicamente inactivos, obesos, con una salud de regular a pobre, dificultades con el autocuidado y menor participación social en comparación a personas sin esta discapacidad ni depresión ${ }^{33}$.

\section{Comorbilidades psicológicas en PcD auditiva}

Las $\mathrm{PcD}$ auditiva en general reportan mayores comorbilidades psicológicas, como una mayor incidencia de demencia ${ }^{34,35}$. También, reportan mayores índices de depresión ${ }^{28,36}$ y de otras disfunciones mentales ${ }^{31}$. Se ha encontrado una asociación independiente entre algún desorden o deterioro cognitivo acelerado y la discapacidad auditiva $^{37-40}$. A su vez, niños y adolescentes con discapacidad auditiva reportan mayor riesgo de depresión, trastorno oposicionista desafiante y otras psicopatologías, así como mayores dificultades emocionales y de comportamiento que sus contrapartes sin esta discapacidad ${ }^{41,42}$. También se reporta que los adolescentes sordos o con hipoacusia reportan mayores de problemas de salud mental ${ }^{43}$.

\section{Calidad de vida}

Se ha reportado que la discapacidad visual incide negativamente en la calidad de vida de las personas ancianas ${ }^{44}$. Además, su calidad de vida se ve afectada por el estado de su salud mental ${ }^{45}$. La depresión es el único factor que se ha encontrado asociado con la calidad de vida de las PcD visual $^{45,46}$. Por ello, los adultos con ceguera se enfrentan a desigualdades en salud que pueden disminuir su calidad de vida si no reciben una intervención oportuna dirigida al manejo del sedentarismo y la angustia psicológica que considere su discapacidad ${ }^{9}$. La discapacidad auditiva tiene un impacto negativo en la calidad de vida, especialmente respecto a los aspectos sociales y emocionales de la comunicación en los adultos ${ }^{40}, y$ en los aspectos educacionales y sociales en niños ${ }^{47}$.

\section{Alfabetismo en salud}

El $48 \%$ de las PcD auditiva reporta un bajo alfabetismo en salud, y son 6,9 veces más propensos que sus contrapartes oyentes a tener un alfabetismo en salud inadecuado ${ }^{48,49}$.

Además, el conocimiento respecto a enfermedad cardiovascular en población adolescente y adulta Sorda es considerablemente menor que el resto de la población ${ }^{50-52}$. Finalmente, los estudiantes universitarios Sordos e hipoacúsicos reportan valores significativamente menores respecto a conocimiento del VIH/SIDA frente a sus contrapartes oyentes ${ }^{53,54}$.

\section{Acceso a atención sanitaria}

La mayoría de las PcD sensorial reportan dificultad para conseguir una cita con un médico, barreras en la comunicación con el tratante y personal de oficina, falta de sensibilización y formación de los prestadores de salud en temas de discapacidad ${ }^{12,26,55-59}$.

Otra importante barrera reportada es frente al acceso a información en temas de salud en los distintos niveles de prevención. Las PcD auditiva no tienen acceso a la información sobre temas en salud transmitidos por radio o televisión ${ }^{57,60}$. Por otro lado, las $\mathrm{PcD}$ visual tampoco tienen acceso a esta información cuando es transmitida a través de pancartas, panfletos o en diarios murales ${ }^{5,56}$.

Específicamente, las $\mathrm{PcD}$ auditiva advierten la barrera de acceso frente a la solicitud de citas médicas vía teléfono (generalmente la única vía disponible $)^{63,70}$, barreras en la comunicación con el personal de oficina ${ }^{13,60,61}$, desconocimiento de la Lengua de Señas ${ }^{57,60-65}$, percepciones de discriminación, desconfianza ${ }^{57,61}$, maltrato ${ }^{65}$, frustración ${ }^{57,61}$ y miedo frente al personal de salud ${ }^{57,61,64}$. Además, advierten que para la entrega de información deben depender de la lectura labiofacial y notas escritas, de las cuales ninguna permite una comunicación adecuada entre el profesional de salud y el paciente Sordo ${ }^{57,60,61,63,65,66}$. Para subsanar esta situación, se utiliza a un familiar como interprete, lo que no es una solución pertinente y coarta la autonomía del paciente ${ }^{57,61-65}$. También advierten la utilización de párrafos extensos y la utilización de un lenguaje muy técnico por parte de los profesionales de la salud ${ }^{57,60,61,65,66}$. Las PcD auditiva que utilizan lenguaje oral solicitan una mayor concientización acerca la sordera por parte de los profesionales de la salud ${ }^{60,67}$.

Las PcD visual también reportan dificultad en la comunicación con médicos generales y especialistas ${ }^{59,68,69}$, refieren dificultad para encontrar los botones para abrir puertas de ingreso a centros médicos ${ }^{69}$, advierten faltas de respeto por parte del personal de salud ${ }^{70} \mathrm{e}$ impaciencia por parte del personal de oficina ${ }^{59}$, infantilización ${ }^{68}$, el dirigirse 
a su acompañante y no a la $\mathrm{PcD}$ visual ${ }^{70}$, barreras físicas para ingresar y salir de los entornos médicos y navegar durante todo el proceso de atención sanitario $^{59,69}$. También refieren dificultad en saber si se encuentra alguien en recepción y con las firmas en pantallas digitales ${ }^{69}$.

Además, advierten que la información respecto a los cuidados a seguir o la posología de los medicamentos es entregada por escrito, lo que no es accesible para ellos ${ }^{55,59,68-70}$. También reportan falta de medios alternativos como el uso de grabaciones de audio y/o Braille y/o letra grande ${ }^{5,59,69,70}$. Esto se concreta en la pérdida de citas médicas por no poder leerlas ${ }^{74}$, o problemas en el reconocimiento de los medicamentos ${ }^{70-72}$. El $30 \%$ de estos pacientes reporta dificultad para leer las etiquetas y posología de fármacos ${ }^{55}$, mientras que $44 \%$ de las mujeres con discapacidad visual colocaron incorrectamente los medicamentos en sus cajas respectivas. Lo anterior pone riesgo la adherencia al tratamiento y con ello la recuperación de su salud ${ }^{73}$.

De acuerdo a datos de Taiwán, la utilización general del examen de salud ofrecido solo alcanza al $16 \%$ de las PcD visual ${ }^{10}$, en los Estados Unidos de Norteamérica las $\mathrm{PcD}$ visual son más propensas a no poseer una fuente de cuidado habitual y experimentan mayores necesidades insatisfechas en referencia a sus contrapartes con otra discapacidad $^{56}$.

\section{Formación profesional sanitario.}

La falta de la disponibilidad de personal sanitario especializado en la atención de PcD visual y auditiva en atención primaria se debe a la ausencia de (o inadecuada) formación dentro de sus programas de estudios en temas de discapacidad ${ }^{74-76}$. En general, el profesional sanitario no se siente capacitado para atender las necesidades de las PcD, reportan menor probabilidad de entregar atención a las $\mathrm{PcD}$ de origen visual y auditiva y evidencian que son los más complejos de atender debido a las barreras de comunicación, siendo mayor al tratar con pacientes con discapacidad auditiva ${ }^{57,77-79}$. Esta situación obstaculiza la entrega de los servicios sanitarios, poniendo en riesgo la recuperación de la salud de estos pacientes.

Se debe comprender la realidad del día a día que están expuestos los pacientes con discapacidad visual y auditiva, y de esa manera poder crear el vínculo médico-paciente, mejorar la confianza, la satisfacción usuaria, el uso de medidas preventivas, asistencia a controles y con ello sus condiciones de salud ${ }^{57,80}$. A nivel internacional se han desarrollado exitosos programas para la atención de personas con discapacidad visual y auditiva en estudiantes de medicina ${ }^{80-85}$, odontología ${ }^{85-87}$, químico-farmacéuticos ${ }^{84,88}$ y profesionales de la salud en general ${ }^{85,89,90}$. Estos programas han resultado en alumnos con mejores actitudes frente a estos pacientes y con la percepción de sentirse capacitados para atender sus necesidades ${ }^{57,83,88,89}$.

\section{Iniciativas y centros de salud especializados}

En Escocia, el "Sensory Support Centre WISC" presenta un alto nivel de satisfacción en cuanto al servicio recibido, valorándose particularmente la forma en que el personal del WISC demuestra conocimiento y empatía con los retos que experimentan debido a la discapacidad visual o auditiva, mejorando su calidad de vida ${ }^{91}$. En Austria, unido a los hospitales, se ha desarrollado "Health Center for the Deaf" donde se entrega un acceso real a la salud, a través de personal competente y capaces de comunicarse en LS u otros medios de comunicación, de acuerdo a las necesidades del paciente ${ }^{57}$. En Francia existe un servicio ambulatorio para la atención primaria de personas Sordas el cual también ha sido favorable para dicha población ${ }^{92}$.

En nuestro país, gracias al financiamiento del Servicio Nacional de la Discapacidad, se ha desarrollado de un software para teléfonos móviles dirigido a la eliminación de los problemas de comunicación que experimentan las personas Sordas durante la atención dental en Centros de Atención Primaria, a través de videos informativos en LS, facilitando el diagnóstico, tratamiento y con ello, mejorando la satisfacción usuaria de los pacientes ${ }^{93}$.

En Brasil, se realizó un pilotaje utilizando videos en LS brasileña (Libras) para identificar factores de riesgo de diabetes o hipertensión asociados a los hábitos alimentarios de PcD auditiva con una gran aceptación por parte de las enfermeras y los pacientes con discapacidad auditiva ${ }^{94}$.

\section{Discusión}

A pesar que en 2010 entró en vigencia la Ley sobre Discapacidad en Chile, donde se indica el diseño de espacios transitables, el empleo del sistema Braille para $\mathrm{PcD}$ visual y reconoce la Lengua 
de Señas como el medio de comunicación natural de la comunidad Sorda, aún no se han establecido como medidas de asistencias sanitarias la obligatoriedad de poseer profesionales del área de la salud con las capacitaciones pertinentes para prestar estos servicios en los establecimientos de salud públicos o privados ${ }^{22}$.

La falta de información en salud y la insatisfacción usuaria de esta población sumado a la falta de capacitación y concientización del personal sanitario acarrean varios problemas para las $\mathrm{PcD}$ sensorial. Lo anterior, implica un desconocimiento de su propio estado de salud, los tratamientos o cuidados a seguir, un menor uso de los servicios de salud en los distintos niveles de prevención y en sus distintas áreas, lo que generaría una mayor incidencia de comorbilidades y un mayor gasto en salud $^{10,55,57,80,95}$.

A pesar que se han reportado exitosos programas de capacitación en personal sanitario a nivel internacional ${ }^{57,80-90}$, en Chile aún no se le ha entregado la importancia debida, por lo que no existe formación establecida en pre y postgrado, a excepción de asignaturas electivas o Diplomados, en donde se intenta abarcar todas las discapacidades en un corto período de tiempo.

Aunque existe un creciente interés por cerrar la brecha de calidad en atención en salud entre personas con y sin discapacidad, existe escasa información sobre la experiencia durante la atención en salud de las $\mathrm{PcD}$ auditiva y visual, así como de sus necesidades de salud respectivas, reportando una insatisfacción usuaria ${ }^{56,80}$. Por otro lado, es aún más escasa la información disponible sobre la percepción del profesional sanitario acerca de su capacidad para brindar sus servicios de manera adecuada a esta población ${ }^{79}$.

Este es un problema de salud pública con escasa visibilidad en nuestro país. Sin embargo, a pesar de la limitada información disponible respecto a las brechas específicas en la atención en salud de esta población, se hacen evidentes las inequidades que enfrentan en cuanto a acceso e información. Estas, al no ser enfrentadas oportunamente, disminuyen aún más la calidad de vida de esta población ${ }^{9,96}$.

El abordaje en Chile hacia las $\mathrm{PcD}$ auditiva y visual han sido dirigidos netamente a la rehabilitación de las $\mathrm{PcD}$, a través de las políticas GES de cataratas, implantes cocleares, audífonos, trasplantes de córnea, etc. Sin embargo, se requiere complementar con un abordaje dirigido a la formación de profesional sanitario, pues se debe aclarar que no todas las $\mathrm{PcD}$ visual o auditiva son candidatos a estas intervenciones y que en muchos casos no es posible eliminar la deficiencia por completo.

Dado lo anterior, es nuestro deber como profesionales de la salud recordar que la Convención de los Derechos de las Personas con Discapacidad (CRPD) ratificada por Chile y más de 100 países, reconoce a las $\mathrm{PcD}$ como sujetos de derecho, protegiendo su dignidad y reconocimiento como un individuo más ${ }^{23,97}$. Esto cobra especial importancia en el caso de las $\mathrm{PcD}$ auditiva y visual que no son candidatos para tratarse con actuales tecnologías médicas.

Los profesionales de la salud debemos reconocer que las $\mathrm{PcD}$ auditiva o visual no siempre necesitar ser "reparados" o depender de otros para su autocuidado. Como refuerza la CRPD, son ciudadanos con derechos humanos, incluyendo el derecho a la atención en salud, a la rehabilitación, el derecho a vivir en comunidad y el derecho a tener voz respecto a su propio tratamiento. Por ello, debemos superar un modelo médico que reduce la discapacidad a un déficit individual y que puede legitimar el paternalismo médico ${ }^{23,97}$. Esto nos llevará a un modelo social que enfrente y dé solución a las barreras y prejuicios que excluyen a las PcD.

Dentro de las limitaciones de esta revisión de la literatura se encuentra la ausencia de estudios prospectivos, en donde se pueda establecer causalidad entre la discapacidad y las comorbilidades reportadas. Sin embargo, esta revisión no busca causalidad, sino caracterizar a las $\mathrm{PcD}$ auditiva o visual y su experiencia con los equipos de salud.

Otra limitación de esta revisión es que debido a que la mayoría de la literatura no hace distinciones entre la naturaleza y característica de la discapacidad sensorial, no es posible describir por subgrupos, como, por ejemplo, las barreras que podría percibir una persona legalmente ciega con una con baja visión, o una persona ciega congénita y una adquirida en la adultez. Lo mismo sucede con la discapacidad auditiva, en donde en la mayoría no se hace distinciones respecto al grado de la deficiencia y el momento de adquisición. El grado de la deficiencia y el momento de la adquisición son importantes, pues una persona con presbiacusia tuvo acceso durante su vida al lenguaje oral, a escribir, leer, acceso a la información. En cambio, una persona con sordera congénita, no ha tenido 
el mismo acceso debido a las barreras previamente reportadas.

Se hace necesario iniciar una línea de investigación nacional que aborde las experiencias de atención sanitaria de las $\mathrm{PcD}$ visual y auditiva, a través de un enfoque cualitativo, lo que permitirá conocer la multidimensionalidad de las percepciones de esta población, esto permitirá informar no solo a las autoridades de las necesidades reales y actuales de esta población, también permitirá plantear posibles vías de mejoramiento que deberán ser evaluadas a futuro.

\section{Conclusiones}

La literatura muestra que las $\mathrm{PcD}$ visual y auditiva reportan mayores tasas de diabetes, enfermedades cardíacas, depresión, entre otras. Sin embargo, la evidencia no muestra causalidad entre la discapacidad auditiva y visual y dichas comorbilidades, sólo asociación.

Esta población también reporta problemas de acceso a los servicios sanitarios debido a la falta de la disponibilidad de personal sanitario especializado, lo que a su vez se debe en parte a la ausencia de formación dentro de sus programas de estudios. Específicamente las PcD visual refieren barreras físicas y las $\mathrm{PcD}$ auditiva barreras comunicacionales.

Existe extensa literatura internacional que aborda estas temáticas, sin embargo, no se reportan estudios nacionales, por lo que se hace necesario generar evidencia con pertinencia local para atender las necesidades de la población con discapacidad sensorial en nuestro país.

\section{Referencias}

1. World Health Organization. Deafness and hearing loss. Fact sheet. 2019. https://www.who.int/news-room/factsheets/detail/deafness-and-hearing-loss.

2. World Health Organization. Visual impairment and blindness. Fact sheet. 2018. https://www.who.int/newsroom/fact-sheets/detail/blindness-and-visual-impairment.

3. Ministerio de Desarrollo Social. Segundo Estudio Nacional de la Discapacidad. 2015. https://www.senadis.gob. cl/pag/355/1197/ii_estudio_nacional_de_discapacidad

4. Murray CJ, Vos T, Lozano R, Naghavi M, Flaxman AD,
Michaud C, et al. Disability-adjusted life years (DALYs) for 291 diseases and injuries in 21 regions, 1990-2010: a systematic analysis for the Global Burden of Disease Study 2010. Lancet 2013; 380 (9859): 2197-223.

5. Stevens G, Flaxman S, Brunskill E, Mascarenhas M, Mathers CD, Finucane M. Global and regional hearing impairment prevalence: an analysis of 42 studies in 29 countries. Eur J Public Health. 2013; 23 (1): 146-52.

6. World Health Organization. Global data on visual impairments 2010. Geneva: World Health Organization Organization.2012. https://www.who.int/blindness/ GLOBALDATAFINALforweb.pdf

7. Bourne RR, Stevens GA, White RA, Smith JL, Flaxman SR, Price H. Causes of vision loss worldwide, 1990-2010: a systematic analysis for the Global Burden of Disease Study 2010. Lancet 2013;1 (6): e339-49.

8. Blanchfield BB, Feldman JJ, Dunbar JL, Gardner EN. The severely to profoundly hearing-impaired population in the United States: Prevalence estimates and demographics. J Am Acad Audiol 2010; 12 (4): 183-9.

9. Jones GC, Crews JE, Danielson ML. Health risk profile for older adults with blindness: an application of the International Classification of Functioning, Disability, and Health framework. Ophthalmic epidemiol 2010; 17 (6): 400-10.

10. Hsu YH, Tsai WC, Kung PT. Health examination utilization in the visually disabled population in han: a nationwide population-based study. BMC Health Serv Res 2013; 13 (1): 509.

11. Mithen J, Aitken Z, Ziersch A, Kavanagh AM. Inequalities in social capital and health between people with and without disabilities. Soc Sci Med 2015; 126: 26-35.

12. Maart S, Jelsma J. Disability and access to health care-a community based descriptive study. Disabil Rehabil 2014; 36 (18): 1489-93.

13. Campos V. Barriers deaf patients face when receiving dental treatment. J Oral Res 2016; 5 (4): 144-5.

14. Shankweiler D, Fowler CA. Seeking a reading machine for the blind and discovering the speech code. Hist Psychol 2015; 18 (1): 78.

15. Ladd P. Understanding deaf culture: In search of deafhood. Multilingual Matters. 2003.

16. Herrera V. Habilidad lingüística y fracaso lector en los estudiantes sordos. Estudios pedagógicos 2005; 31 (2): 121-35.

17. Qi S, Mitchell RE. Large-scale academic achievement testing of deaf and hard-of-hearing students: Past, present, and future. Journal of Deaf Studies and Deaf Education 2012; 17: 1-18.

18. Lučić B, Ostrogonac S, Vujnović Sedlar N, Sečujski M. Educational applications for blind and partially sighted 
pupils based on speech technologies for Serbian. The Scientific World Journal, 2015.

19. Virgili G, Acosta R, Grover LL, Bentley SA, Giacomelli G. Reading aids for adults with low vision. Cochrane Database Syst Rev 2013; (10): CD003303.

20. Šepić B, Ghanem A, Vogel S. BrailleEasy: One-handed Braille Keyboard for Smartphones. Stud Health Technol Inform 2015; 217: 1030-5.

21. Jiménez J, Olea J, Torres J, Alonso I, Harder D, Fischer $\mathrm{K}$. Biography of louis braille and invention of the braille alphabet. Surv Ophthalmol. 2009; 54 (1): 142-9.

22. Ley $\mathrm{N}^{\circ}$ 20.422. Establece normas sobre igualdad de oportunidades e inclusión social de personas con discapacidad. Diario Oficial de la República de Chile, Santiago, Chile, 10 de febrero de 2010. http://bcn.cl/1uvqg.

23. United Nations; United Nations Human Rights Office of the High Commissioner. The Convention on the Rights of Persons with Disabilities. New York and Geneva: United Nations Publication; 2014.

24. Armstrong TW, Surya S, Elliott TR, Brossart DF, Burdine JN. Depression and health-related quality of life among persons with sensory disabilities in a health professional shortage area. Rehabil Psychol. 2016; 61 (3): 240-50.

25. Heine C, Browning CJ. Communication and psychosocial consequences of sensory loss in older adults: overview and rehabilitation directions. Disabil Rehabil. 2002; 24 (15): 763-73.

26. Court H, McLean G, Guthrie B, Mercer SW, Smith DJ. Visual impairment is associated with physical and mental comorbidities in older adults: a cross-sectional study. BMC Med 2014; 12: 181.

27. Crews JE, Jones GC, Kim JH. Double jeopardy: the effects of comorbid conditions among older people with vision loss. J Vis Impair Blind 2006; 100: 824.

28. Emond A, Ridd M, Sutherland H, Allsop L, Alexander A, Kyle J. The current health of the signing Deaf community in the UK compared with the general population: a cross-sectional study. BMJ Open 2015; 5 (1): e006668.

29. McKee MM, Stransky ML, Reichard A. Hearing loss and associated medical conditions among individuals 65 years and older. Disabil Health J 2018; 11 (1): 122-5.

30. Mann JR, Zhou L, McKee M, McDermott S. Children with hearing loss and increased risk of injury. Ann Fam Med 2007; 5 (6): 528-33.

31. Genther DJ, Frick KD, Chen D, Betz J, Lin FR. Association of hearing loss with hospitalization and burden of disease in older adults. JAMA 2013; 309 (22): 2322-4.

32. Ribeiro MV, Hasten-Reiter Júnior HN, Ribeiro EA, Jucá MJ, Barbosa FT, Sousa-Rodrigues CF. Association between visual impairment and depression in the elder- ly: a systematic review. Arq Bras Oftalmol 2015; 78 (3): 197-201.

33. Jones GC, Rovner BW, Crews JE, Danielson ML. Effects of depressive symptoms on health behavior practices among older adults with vision loss. Rehabil Psychol 2009; 54 (2): 164-72.

34. Thomson RS, Auduong P, Miller AT, Gurgel RK. Hearing loss as a risk factor for dementia: a systematic review. Laryngoscope Investig Otolaryngol 2017; 2 (2): 69-79.

35. Ford AH, Hankey GJ, Yeap BB, Golledge J, Flicker L, Almeida OP. Hearing loss and the risk of dementia in later life. Maturitas 2018; 112: 1-11.

36. Fellinger J, Holzinger D, Pollard R. Mental health of deaf people. Lancet 2012; 379 (9820): 1037-44.

37. Lin FR, Yaffe K, Xia J, Xue QL, Harris TB, Purchase-Helzner E, et al; Health ABC Study Group. Hearing loss and cognitive decline in older adults. JAMA Intern Med 2013;173(4):293-9.

38. Taljaard DS, Olaithe M, Brennan-Jones CG, Eikelboom $\mathrm{RH}$, Bucks RS. The relationship between hearing impairment and cognitive function: a meta-analysis in adults. Clin Otolaryngol 2016; 41 (6): 718-29.

39. Zheng Y, Fan S, Liao W, Fang W, Xiao S, Liu J. Hearing impairment and risk of Alzheimer's disease: a meta-analysis of prospective cohort studies. Neurol Sci 2017; 38 (2): 233-9.

40. Bainbridge KE, Wallhagen MI. Hearing loss in an aging American population: extent, impact, and management. Annu Rev Public Health 2014; 35: 139-52.

41. Stevenson J, Kreppner J, Pimperton H, Worsfold S, Kennedy C. Emotional and behavioural difficulties in children and adolescents with hearing impairment: A systematic review and meta-analysis. Eur Child Adolesc Psychiatry 2015; 24 (5): 477-96.

42. Theunissen SC, Rieffe C, Netten AP, Briaire JJ, Soede W, Schoones JW, et al. Psychopathology and its risk and protective factors in hearing-impaired children and adolescents: a systematic review. JAMA Pediatr 2014; 168 (2): 170-7.

43. Brown PM, Cornes A. Mental health of deaf and hardof-hearing adolescents: what the students say. J Deaf Stud Deaf Educ 2015; 20 (1): 75-81.

44. Kwon HJ, Kim JS, Kim YJ, Kwon SJ, Yu JN. Sensory impairment and health-related quality of life. Iran J Public Health 2015; 44 (6): 772-82.

45. Crewe JM, Morlet N, Morgan WH, Spilsbury K, Mukh$\operatorname{tar}$ A, Clark A, et al. Quality of life of the most severely vision-impaired. Clin Exp Ophthalmol 2011; 39 (4): 336-43.

46. Crews JE, Chou CF, Zhang X, Zack MM, Saaddine JB. 
Health-related quality of life among people aged $\geq 65$ years with self-reported visual impairment: Findings from the 2006-2010 behavioral risk factor surveillance system. Ophthalmic Epidemiol. 2014; 21 (5): 287-96.

47. Roland L, Fischer C, Tran K, Rachakonda T, Kallogjeri D, Lieu JE. Quality of life in children with hearing impairment: systematic review and meta-analysis. Otolaryngol Head Neck Surg 2016; 155 (2): 208-19.

48. McKee MM, Paasche-Orlow MK, Winters PC, Fiscella $\mathrm{K}$, Zazove $\mathrm{P}$, Sen A, et al. Assessing health literacy in deaf american sign language users. J Health Commun 2015; 20 Suppl 2: 92-100.

49. McKee M, Schlehofer D, Cuculick J, Starr M, Smith S, Chin NP. Perceptions of cardiovascular health in an underserved community of deaf adults using American Sign Language. Disabil Health J 2011; 4 (3): 192-7.

50. Margellos-Anast H, Estarziau M, Kaufman G. Cardiovascular disease knowledge among culturally Deaf patients in Chicago. Prev Med 2006; 42 (3): 235-9.

51. Smith SR, Samar VJ. Dimensions of deaf/hard-of-hearing and hearing adolescents' health literacy and health knowledge. J Health Commun 2016; 21 (sup2): 141-54.

52. Smith SR, Kushalnagar P, Hauser PC. Deaf adolescents' learning of cardiovascular health information: sources and access challenges. J Deaf Stud Deaf Educ 2015; 20 (4): 408-18.

53. Heuttel KL, Rothstein WG. HIV/AIDS knowledge and information sources among deaf and hearing college students. Am Ann Deaf 2001; 146 (3): 280-6.

54. Groce NE, Yousafzai AK, van der Maas F. HIV/AIDS and disability: Differences in HIV/AIDS knowledge between deaf and hearing people in Nigeria. Disabil Rehabil 2007; 29 (5): 367-71.

55. Beverley CA, Bath PA, Booth A.. Health information needs of visually impaired people: a systematic review of the literature. Health Soc Care Community 2004; 12 (1): 1-24.

56. Horner-Johnson W, Dobbertin K, Lee JC, Andresen EM; Expert Panel on Disability and Health Disparities. Disparities in health care access and receipt of preventive services by disability type: analysis of the medical expenditure panel survey. Health Serv Res 2014; 49 (6): 1980-99.

57. Kuenburg A, Fellinger P, Fellinger J. Health care access among deaf people. J Deaf Stud Deaf Educ 2016; 21 (1): $1-10$.

58. Merten JW, Pomeranz JL, King JL, Moorhouse M, Wynn RD. Barriers to cancer screening for people with disabilities: a literature review. Disabil Health J 2015; 8 (1): 9-16.

59. Sharts-Hopko NC, Smeltzer S, Ott BB, Zimmerman V,
Duffin J. Healthcare experiences of women with visual impairment. Clin Nurse Spec 2010; 24 (3): 149-53.

60. Emond A, Ridd M, Sutherland H, Allsop L, Alexander A, Kyle J. Access to primary care affects the health of Deaf people. Br J Gen Pract 2015; 65 (631): 95-6.

61. Iezzoni LI, O'Day BL, Killeen M, Harker H. Communicating about health care: observations from persons who are deaf or hard of hearing. Ann Intern Med 2004; 140 (5): 356-62.

62. da Silva Bentes IM, Vidal ECF, Maia ER. Deaf person's perception on health care in a midsize city: an descriptive-exploratory study. Online Brazilian Journal of Nursing 2011; 10 (1).

63. Aragão JS, Magalhães IMO, Coura AS, Silva AFR, Cruz, GKP, França ISXD. Access and communication of deaf adults: a voice silenced in health services. J Res Fundam Care online 2014; 6 (1): 1-7.

64. Kritzinger J, Schneider M, Swartz L, Braathen SH. "I just answer 'yes' to everything they say": Access to health care for deaf people in Worcester, South Africa and the politics of exclusion. Patient Educ Couns 2014; 94 (3): 379-83.

65. Gichane MW, Heap M, Fontes M, London L. "They must understand we are people": Pregnancy and maternity service use among signing Deaf women in Cape Town. Disabil Health J 2017; 10 (3): 434-9.

66. Oliveira YCA, Coura AS, Costa GMC, de França ISX. Communication between health professionals-deaf people: an integrative review. Journal of Nursing UFPE on line 2015; 9 (2): 957-64.

67. Middleton A, Turner GH, Bitner-Glindzicz M, Lewis P, Richards M, Clarke A, et al. Preferences for communication in clinic from deaf people: A cross-sectional study. J Eval Clin Pract 2010; 16 (4): 811-7.

68. Smeltzer SC, Sharts-Hopko NC, Ott BB, Zimmerman V, Duffin J. Perspectives of women with disabilities on reaching those who are hard to reach. J Neurosci Nurs 2007; 39 (3): 163-71.

69. Cupples ME, Hart PM, Johnston A, Jackson AJ. Improving healthcare access for people with visual impairment and blindness. BMJ 2012; 344: e542.

70. O'Day BL, Killeen M, Iezzoni LI.. Improving health care experiences of persons who are blind or have low vision: suggestions from focus groups. Am J Med Qual 2004; 19 (5): 193-200.

71. Windham BG, Griswold ME, Fried LP, Rubin GS, Xue QL, Carlson MC. Impaired vision and the ability to take medications. J Am Geriatr Soc 2005; 53 (7): 1179-90.

72. McCann RM, Jackson AJ, Stevenson M, Dempster M, McElnay JC, Cupples ME. Help needed in medication self-management for people with visual impairment: 
case-control study. Br J Gen Pract 2012; 62 (601): e5307.

73. Smith M, Bailey T. Identifying solutions to medication adherence in the visually impaired elderly. Consult Pharm 2014; 29 (2): 131-4.

74. Smeltzer SC, Blunt E, Marozsan H, Wetzel-Effinger L. Inclusion of disability-related content in nurse practitioner curricula. J Am Assoc Nurse Pract 2015; 27 (4): 213-21.

75. Ahmad MS, Razak IA, Borromeo GL. Special Needs Dentistry: perception, attitudes and educational experience of Malaysian dental students. Eur J Dent Educ 2015; 19 (1): 44-52.

76. Dellavia C, Allievi C, Ottolina P, Sforza C. Special care dentistry for people with intellectual disability in dental education: an Italian experience. Eur J Dent Educ 2009; 13 (4): 218-22.

77. Merten JW, Pomeranz JL, King JL, Moorhouse M, Wynn RD. Barriers to cancer screening for people with disabilities: a literature review. Disabil Health J 2015; 8 (1): 9-16.

78. Suhani RD, Suhani MF, Muntean A, Mesaros M, Badea ME. Ethical dilemmas concerning the dental treatment of patients with hearing impairment. Revista Romana de Bioetica 2015; 13 (3).

79. Bachman SS, Vedrani M, Drainoni ML, Tobias C, Maisels L. Provider perceptions of their capacity to offer accessible health care for people with disabilities. J Disabil Policy Stud 2006; 17 (3): 130-6.

80. Sharby N, Martire K, Iversen MD. Decreasing health disparities for people with disabilities through improved communication strategies and awareness. Int J Environ Res Public Health 2015; 12 (3): 3301-16.

81. Lock E. A workshop for medical students on deafness and hearing impairments. Acad Med 2003; 78 (12): 1229-34.

82. Hoang L, LaHousse SF, Nakaji MC, Sadler GR. Assessing deaf cultural competency of physicians and medical students. J Cancer Educ 2011; 26 (1): 175-82.

83. Thew D, Smith SR, Chang C, Starr M. The deaf strong hospital program: a model of diversity and inclusion training for first-year medical students. Acad Med 2012; 87 (11): 1496-500.

84. Van Winkle LJ, Fjortoft N, Hojat M. Impact of a workshop about aging on the empathy scores of pharmacy and medical students. Am J Pharm Educ 2012; 76 (1): 9.

85. Oliveira YCA, Costa GMC, Coura AS, Cartaxo RO,
França ISX. Brazilian sign language in the training of nursing, physiotherapy and dentistry professionals in the state of Paraíba, Brazil. Interface-Comunicação, Saúde, Educação 2012; 16 (43): 995-1008.

86. Jones T, Cumberbatch K. Sign language in dental education-A new nexus. Eur J Dent Educ 2018; 22 (3): 143-50.

87. Santos Pérez Y, Novoa López AM. Valoraciones de futuros estomatólogos sobre su formación para la atención al paciente sordo, La Habana, 2016. Revista Habanera de Ciencias Médicas 2017; 16 (2): 280-94.

88. Mathews JL, Parkhill AL, Schlehofer DA, Starr MJ, Barnett S. Role-reversal exercise with deaf strong hospital to teach communication competency and cultural awareness. Am J Pharm Educ 2011; 75 (3): 53.

89. Castro SS, Rowe M, Andrade LF, Cyrino EG. Developing competencies among health professions students related to the care of people with disabilities: a pilot study. Interface-Comunicação, Saúde, Educação. 2017.

90. Robey KL, Minihan PM, Long-Bellil LM, Hahn JE, Reiss JG, Eddey GE; Alliance for Disability in Health Care Education. Teaching health care students about disability within a cultural competency context. Disabil Health J 2013; 6 (4): 271-9.

91. Smith A, Shepherd A, Jepson R, Mackay S. The impact of a support centre for people with sensory impairment living in rural Scotland. Prim Health Care Res Dev 2016; 17 (02): 138-48.

92. Amoros T, Bonnefond H, Martínez C, Charles R. A dedicated ambulatory system for the primary healthcare of the deaf people. Sante Publique 2014; 26 (2): 205-15.

93. Campos V, Cartes-Velásquez R, Bancalari C. Development of an App for the dental care of Deaf people: Odontoseñas (2019). Universal Access in the Information Society. DOI: 10.1007/s10209-018-0637-1.

94. Rodrigues SCM, Damião GC. Virtual Environment: assistance in nursing care for the deaf based on the protocol of Primary Care. Revista da Escola de Enfermagem da USP 2014; 48 (4): 731-8.

95. Steinberg AG, Wiggins EA, Barmada $\mathrm{CH}$, Sullivan VJ. Deaf women: Experiences and perceptions of healthcare system access. J Womens Health (Larchmt). 2002; 11 (8): 729-41.

96. Peterson-Besse JJ, O’Brien MS, Walsh ES, Monroe-Gulick A, White G, Drum CE. Clinical preventive service use disparities among subgroups of people with disabilities: A scoping review. Disabil Health J 2014; 7 (4): 373-93. 